\title{
Cloning and functional analysis of the promoter of the sesquiterpene synthase gene ASS1 in Aquilaria sinensis
}

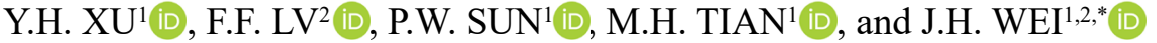 \\ ${ }^{1}$ Key Laboratory of Bioactive Substances and Resources Utilization of Chinese Herbal Medicine, Ministry of \\ Education; Institute of Medicinal Plant Development, Chinese Academy of Medical Sciences and Peking Union \\ Medical College, Beijing 100193, P.R. China \\ ${ }^{2}$ Hainan Branch of the Institute of Medicinal Plant Development, Chinese Academy of Medical Sciences and Peking \\ Union Medical College, Haikou 570311, P.R. China
}

*Corresponding author: E-mail: wjianh@263.net

\begin{abstract}
Agarwood, the resin part of Aquilaria spp., is valued in medicine, perfumes, and incense. The most important components of agarwood are sesquiterpenes, which are produced only when a healthy tree is wounded. Agarwood sesquiterpene synthase 1 (ASS1) is one of key enzymes responsible for the biosynthesis of sesquiterpenes in Aquilaria sinensis (Lour.) Gilg, and it is a typical wound-inducible synthase. To elucidate its regulatory mechanism at the transcriptional level, a 978-bp sequence upstream of the translation initiation codon ATG of the promoter for ASS1 was cloned. Computational analysis revealed that this promoter contained many known cis-elements including several defense related transcriptional factor-binding boxes. To functionally validate the promoter, a 5 ' truncated fragment fused with the $\beta$-glucuronidase (GUS) reporter gene was used for generating stable transgenic Arabidopsis plants. The spatial and temporal expression patterns of GUS in transgenic Arabidopsis showed that the promoter of ASS1 was induced by mechanical wound and mainly expressed in vascular bundles. Subcellular localization showed that ASS1 localized in the nucleus and plasma membrane. Here, identification of the ASS1 promoter not only lays a foundation for studying its transcriptional regulation, but also provides clues for studying the synthesis mechanism of agarwood sesquiterpenes.
\end{abstract}

Keywords: agarwood, $\beta$-glucuronidase, onion epidermis, transgenic Arabidopsis plants.

\section{Introduction}

When plants encounter herbivorous insects, microbial pathogens, and other attacks, they produce signal molecules to activate expression of defensive genes. In consequence, they synthesize defensive compounds (toxins and feeding deterrents) to prevent the spread of the damage and to sustain growth and to survive (Turlings et al. 1990, Farmer and Ryan 1992, Agrawal 1998, De Moraes et al. 1998, Arimura et al. 2000, Kessler and Baldwin 2002, Pichersky and Gershenzon 2002, Gomi et al. 2003, Lou et al. 2007, Yamasaki et al. 2007). Some of the induced secondary metabolites are also used by humans as pharmaceuticals, cosmetics, perfumes, dyes, or flavors (He and Giusti 2010, Kroymann 2011, Zhou et al. 2011, Duan et al. 2012). Thus, external wound will be applied in order to produce appropriate responses and useful defensive secondary metabolites. For example, when the healthy Aquilaria trees are wounded, the agarwood is formed, which is a fragment and resinous wood (Itoh et al. 2002, Pojanagaroon and Kaewrak 2005, Persoon 2008) that is widely used in traditional medicines, incense, and perfumes (Kumeta and Ito 2010, Liu et al. 2013, Xu et al. 2013). Aquilaria sinensis (Lour.) Gilg is one of the most important plant resources for producing agarwood in China, and it is also the only certified source for agarwood listed in the China

$\overline{\text { Received }} 27$ April 2020, last revision 25 September 2020, accepted 29 September 2020.

Abbreviations: ASS1 - agarwood sesquiterpene synthase 1; GFP - green fluorescent protein; GUS - $\beta$-glucuronidase; RACE - rapid amplification of cDNA ends.

Acknowledgements: This work was supported by the National Natural Science Foundation of China (81573525), and the Program of CAMS Initiative for Innovative Medicine (CAMS-I2M) (2016-I2M-2-003), and the National Key Research and Development projects (2018YFC1706401).

Conflict of interest: The authors declare that they have no conflict of interest. 
Pharmacopoeia (China pharmacopoeia Committee 2010).

Studies have revealed that sesquiterpene is one of the major bioactive components of agarwood (Hashimoto et al. 1985, Chen et al. 2011, 2012). Like other sesquiterpene phytoalexins, agarwood sesquiterpene accumulates in response to elicitation or wound (Ito et al. 2005, Okudera and Ito 2009, Xu et al. 2016). The first committed step of sesquiterpene biosynthesis in $A$. sinensis is the cyclization of farnesyl diphosphate catalyzed by sesquiterpene synthases, which were cloned independently by Kumeta and Ito (2010) and $\mathrm{Xu}$ et al. (2013). It was found that in healthy Aquilaria calluses or cell culture, expression of sesquiterpene synthase ASS1 is barely detectable, whereas in the wounded tissues or methyl jasmonate treated calluses, its expression increases significantly, and the sesquiterpene compounds raise correspondingly ( $\mathrm{Xu}$ et al. 2013). These results demonstrate that ASS1 is a typical wound-induced gene that is responsible for the formation of agarwood sesquiterpene, and it is primarily regulated at transcriptional level.

Transcriptional regulation plays very important role in the activation or suppression of gene expression, and it is largely controlled through promoters and their contributing cis-acting elements (Zou et al. 2011). Based on the expression type, promoters are often classified as constitutive promoters, spatiotemporal promoters, and inducible promoters (Peremarti et al. 2010, HernandezGarcia and Finer 2014). Inducible promoters are responsive to external physical and chemical stimuli, and endogenous signals (plant hormones). Many typical inducible promoters have been identified in plants and have applications in crop biotechnology. For example, promoters of Arabidopsis Rd29A, rice OsNCED3, and Wsil8 genes are highly-inducible after drought, ABA, and high-salinity treatments (Pellegrineschi et al. 2004, Yi et al. 2011). The defensive promoters, such as rice OsPR10a, barley GER4, and Arabidopsis PDF1.2 and $P R-1$ are highly induced by pathogen infection (Penninckx et al. 1998, Hwang et al. 2008, Himmelbach et al. 2010, Bang et al. 2013). However, there is no report about the promoter of sesquiterpene synthase genes in Aquilaria spp. till now.

To explore the regulation mechanism of sesquiterpene synthase in $A$. sinensis, in the current work, the promoter of ASS1 was cloned and its properties were analyzed by examining $\beta$-glucuronidase (GUS) activity in transgenic Arabidopsis plants harboring ASS1 promoter-GUS gene construct. Our data demonstrated that the ASS1 promoter is a typical wound-inducible promoter and it was highly expressed in vascular bundles. This may be the reason why sesquiterpenes are not formed actively in the healthy $A$. sinensis and they are only produced in the wounded interxylary phloem and xylem rays (Liu et al. 2019). Identification of the ASS1 promoter here can lay the foundation for studying the synthesis mechanism of agarwood sesquiterpenes in $A$. sinensis.

\section{Materials and methods}

Plants and growth conditions: Arabidopsis thaliana L. ecotype Columbia (Col-0) and three-year-old Aquilaria sinensis (Lour.) Gilg (identified by Prof. Jian-He Wei) saplings grown in Yanfeng County, Haikou City, Hainan Province, China, were used in the experiments. Arabidopsis plantlets were grown on Murashige and Skoog medium (Sigma, St. Louis, USA) in a growth chamber at a temperature of $19-20{ }^{\circ} \mathrm{C}$, a 16 -h photoperiod, and an irradiance of $\sim 80 \mu \mathrm{mol}$ (photons) $\mathrm{m}^{-2} \mathrm{~s}^{-1}$ or at about $120 \mu \mathrm{mol}$ (photons) $\mathrm{m}^{-2} \mathrm{~s}^{-1}$ when a compost soil was used.

Genome DNA extraction: The genome DNA was extracted by the cetyltrimethylammonium bromide (CTAB) method. About $3 \mathrm{~g}$ of $A$. sinensis leaves were ground in liquid nitrogen and transferred to a 50$\mathrm{cm}^{3}$ microfuge tube. Ten cubic centimetres-of a $65{ }^{\circ} \mathrm{C}$ preheated cetyltrimethylammonium bromide buffer $[200$ mM Tris-HCl ( $\mathrm{pH} 7.4$ ), $25 \mathrm{mM}$ EDTA, $250 \mathrm{mM} \mathrm{NaCl}$, $0.5 \%(\mathrm{~m} / \mathrm{v})$ sodiumdodecyl sulphate] was added, and leaves were extracted at a $65{ }^{\circ} \mathrm{C}$ water bath for about 45 min. Then, $8 \mathrm{~cm}^{3}$ of saturated phenol/chloroform/isoamyl alcohol (25/24/1, v/v/v) was added, mixed, and extracted for about $30 \mathrm{~min}$. After $5400 \mathrm{~g}$ centrifugation for $15 \mathrm{~min}$, the supernatant was transferred to a clean microfuge tube, and $2 / 3$ volume of ice-cold isopropanol was added. The tube was placed at $-20^{\circ} \mathrm{C}$ for $30 \mathrm{~min}$ and then centrifuged at $10800 \mathrm{~g}$ for $10 \mathrm{~min}$. The supernatant was discarded and the precipitate was washed first with $75 \%(\mathrm{v} / \mathrm{v})$ ethanol and then with $100 \%$ ethanol. Then, the pellet was allowed to dry. Resuspended DNA in $0.7 \mathrm{~cm}^{3}$ of TE buffer $(0.1 \mathrm{M}$ Tris-HCl, pH 7.5, $10 \mathrm{mM}$ EDTA) with added $7 \mathrm{~mm}^{3}$ of RNase was placed at $37^{\circ} \mathrm{C}$ for $1 \mathrm{~h}$, extracted using $5 \mathrm{~cm}^{3}$ of saturated phenol/chloroform/isoamyl alcohol (25/24/1, $\mathrm{v} / \mathrm{v} / \mathrm{v}$ ) for about $5 \mathrm{~min}$, and centrifuged at $10800 \mathrm{~g}$ for $10 \mathrm{~min}$. The supernatant was transferred to a new $1.5-\mathrm{cm}^{3}$ microcentrifuge tube with adding $120 \mathrm{~mm}^{3}$ of TE buffer and $500 \mathrm{~mm}^{3}$ of chloroform/isoamyl alcohol $(24 / 1, \mathrm{v} / \mathrm{v})$, extracted for $5 \mathrm{~min}$, and then centrifuged at $10800 \mathrm{~g}$ for $10 \mathrm{~min}$. The supernatant was again transferred to a new $1.5-\mathrm{cm}^{3}$ microcentrifuge tube, added a $2 / 3$ volume of ice-cold isopropanol, placed at $-20{ }^{\circ} \mathrm{C}$ for $30 \mathrm{~min}$, and then centrifuged at $10800 \mathrm{~g}$ for $10 \mathrm{~min}$. The precipitate was washed with $75 \%(\mathrm{v} / \mathrm{v})$ ethanol and dried at room temperature. The pellet was dissolved in $200-400 \mathrm{~mm}^{3}$ of the TE buffer and stored at $4{ }^{\circ} \mathrm{C}$.

Cloning the promoter of the ASS1 gene from $A$. sinensis: Tail PCR was performed according to the Genome Walking kit (Takara, Tokyo, Japan) using the primer AP1 as a forward primer and primers GSP1: 5'-AGAGAATCCGTGTCCTCGAAGCAACC -3', GSP2: 5'-GTCTCAAAATGATATCCAACGCCCAG-3', or GSP3: 5'-CCTCATGTTTTTCAAGGATGCTGTCG-3' as reverse primers, respectively, for the first, second, and third PCR. The first PCR product was diluted 1000 times and used as template for the second PCR, similarly, the second PCR product was diluted 1000 times and used as template for the third PCR. The third PCR product 
was purified using QIAQuick PCR purification kit (Qiagen, Düsseldorf, Germany) and cloned into pGEM T-easy vector (Promega, Madison, Wisconsin, USA) by T/A cloning and sequencing. A PCR amplification was performed again using specific primers designed based on the sequencing, and the PCR product was sequenced to confirm its correctness.

5 ' rapid amplification of CDNA ends PCR of the ASS1 gene: To confirm the correctness of the promoter sequence, 5' RACE PCR was performed using $10 \times$ Universal Primer $A$ Mix (UPM) as a forward primer and GSP3 as a reverse primer according to the manual of the SMARTer ${ }^{T M}$ RACE cDNA amplication kit (Clontech, San Francisco, USA). The PCR product was purified and cloned into $p G E M$ T-easy vector (Promega, Madison, Wisconsin, USA) by $\mathrm{T} / \mathrm{A}$ cloning and sequencing.

Construction of $\boldsymbol{P}_{A S S I}-\boldsymbol{G U S}$ fusion and generation of transgenic Arabidopsis plants: The promoter fragment was amplified using the primers $\mathrm{GUS}_{731}-\mathrm{F}: 5^{\prime}-\mathrm{CCCAAGCT}$ TGATGCGTATTTGTTCTTTCTTTTCG-3' and GUS-R: 5'- CGGAATTCCAAGAAGTTGGAAGAATGAGTG AGG-3' (corresponding to $-731 \sim 111$ bp DNA sequence) which contain a HindIII and EcoRI site (underlined), and cloned into the binary vector pCAMBIA1391 and digested using the same restriction enzymes. The correct construct was introduced into the GV3101 strain of Agrobacterium tumefaciens and transformed into Arabidopsis wild-type (Col-0) by floral infiltration. Transformed lines were selected on a Murashige and Skoog plate containing $35 \mu \mathrm{g} \mathrm{cm}^{-3}$ hygromycin and $40 \mu \mathrm{g} \mathrm{cm}^{-3}$ carbenicillin and were confirmed by PCR. The T3 generation homozygous plants were used for the analysis of GUS activity.

Histochemical staining GUS: Tissues were stained with 1 mM X-gluc (5-bromo-4-chloro-3-indolyl P-glucuronide) staining solution containing $100 \mathrm{mM} \mathrm{Na}_{3} \mathrm{PO}_{4}(\mathrm{pH} 7.0)$, $0.05 \mathrm{mM}_{3} \quad \mathrm{~K}_{3} \quad\left[\mathrm{Fe}(\mathrm{CN})_{6}\right], \quad 0.05 \quad \mathrm{mM} \quad \mathrm{K}_{4}\left[\mathrm{Fe}(\mathrm{CN})_{6}\right]$, $2 \mathrm{mM}$ EDTA, and $0.1 \%(\mathrm{v} / \mathrm{v})$ Triton $X-100$ at $37{ }^{\circ} \mathrm{C}$ for 12 to $16 \mathrm{~h}$. Tissues were then fixed in $50 \%(\mathrm{v} / \mathrm{v})$ ethanol, $5 \%(\mathrm{~m} / \mathrm{v})$ glacial acetic acid, and $10 \%(\mathrm{v} / \mathrm{v})$ formaldehyde for $2 \mathrm{~h}$. Chlorophyll was removed by extensive washing of the tissue in $70 \%(\mathrm{v} / \mathrm{v})$ ethanol. Tissues for embedding were subsequently dehydrated using solutions of increasing ethanol concentration (twice at $70 \%$ for $8 \mathrm{~h}$, once at $80 \%$ for $30 \mathrm{~min}$, once at $90 \%$ for $30 \mathrm{~min}$, and once at $100 \%$ for $30 \mathrm{~min}$ ).

Subcellular localization of $\boldsymbol{P}_{A S S I}$ in onion epidermis: For the transient expression, $P_{A S S 1}$ was recombined with the vector pAN580 carrying green fluorescent protein (GFP). The sequence of $P_{A S S I}$ was amplified using a forward primer 5'-AAA AGTACTCTCATGTTTTTCAAGGATGCTG TC-3' and a reverse primer 5'-GGACTAGTCAAGAAGT TGGAAGAATGAGTGAGG-3' and cloned into the SacI (5'-end) and SpeI ( 3 '-end) sites upstream of the GFP in the pAN580 vector in which the $35 \mathrm{~S}$ promoter was replaced by the native promoter $P_{A S S 1}$. The construction was confirmed by sequencing and used for transient transformation of onion epidermis via a gold particle bombardment (PDS1000, Bio-Rad, Hercules, California, USA). Fluorescence of GFP was observed after $16 \mathrm{~h}$ of incubation in darkness with a fluorescence microscope (OLYMPUS V-TVO.5XC-3, Tokyo, Japan).

\section{Results}

We have previously reported that $A S S 1$ is a typical inducible gene (Xu et al. 2013). Here, to investigate its transcriptional regulation, a 978 bp upstream fragment was isolated from $A$. sinensis genome DNA through Genome Walking method and named $P_{A S S I}$. Four specially designed degenerate primers with low annealing temperature are provided in the kit. Usually, at least one of them can react with specific primers by thermal asymmetric PCR. After two rounds of PCR, we found that AP1 was the most suitable one (Fig. 1 $A, B$ ). Then, the third nested PCR was performed using the primers GSP3 and AP1 (Fig. 1C) and using the third nested PCR product diluted 1000 times as a template, the specific fragment was successfully obtained (Fig. 1D). After sequencing, we got the specific sequence of promoter fragments. Furthermore, primers were designed according to the specific sequence and PCR was carried

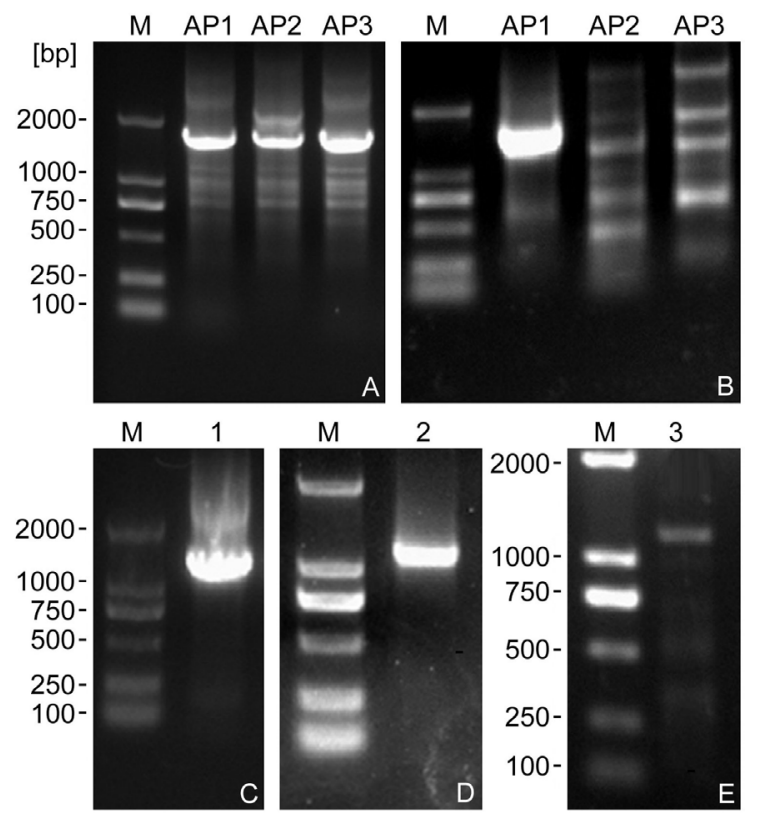

Fig. 1. Cloning and detection of $P_{A S S I}$ using a genome walking kit from Aquilaria sinensis. $A$ - products of the first PCR (AP1, AP2, and AP3 represent products of a specific primer GSP1 with universal primers AP1, AP2, or AP3). $B$ - products of the second PCR (AP1, AP2, and AP3 represent products of a specific primer GSP2 with universal primers AP1, AP2, or AP3). C,D products of the third nest PCR using primers GSP3 and AP1. $E$ - Verification of sequencing obtained in $C$ and $D .1$ - PCR product using GSP3 and AP1 as primers, and genome DNA as template; 2 - PCR product using GSP3 and AP1 as primers, and (1) product diluted 1000 times as template; 3 - PCR product using specific primers and genome DNA as template; $\mathrm{M}$ DL2000 DNA marker. 


\begin{abstract}
GAITCCTCAI GTTTTCAAG GATGCTGTCG AGTATGGTG TGGTGATCTA IAAAITAATA TIGTCCGCCG

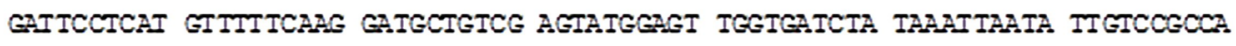

CACCTGAGTC CGTCTIGGA GCA GAATGG ICTGEAGCG CGCTGACCGI CGGCTTGIGA GITGGTTIAC CACCTGAGTC CGTCTTGGA GCAGATGG TCTGGAGGg CGCTGACCGT CGGCTIGTGA GTTGGITTAC

AATICCICTG ICTITITIGI CTITITICTA CTITGGGCAA TGAAGATAGG ACAITTATAI ATCTAIATIT AATTCCTCTG TCTITITIGI CITITTTCTA CTITGGGCAA TGAGAIAGG ACATTTATAI ATCTATATIT

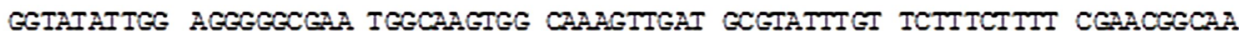

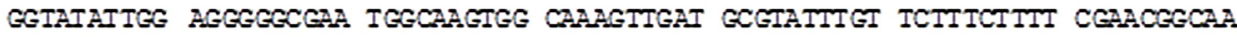

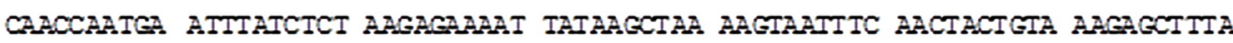
CAACCAATGA ATTTATCTCT AAGAGAAAT TATAAGCTAA AAGTAATTTC AACTACTGTA AAGAGCTTTA

gAAGTACAAA GTTGTIACAG CCAATTACTG ACAGAGCCT TCGGGTTCTC ATACCAITCA AACCATGACC GAGTACAAA GITGITACAE CCAATIACIG ACAGAGCC- -

ACTTTAATT CTIATACAIA ICCGIGTCT AIGAATAAAA AAACATTGG AIAICAICTA CTAACCCACT --_------

TACTGTAAGT TGTTCATCTI IGAAAATAG GCCGTTCTA CTATTCAAAA TICTTCATGI IGTACATGCT IACTGIAAGT IGITCAICTI IGAAACTAG GCCGITTCTA CTAITCAAAA ITCTTCATGI IGIACAIGCT

CTTACGGCTT CTCAAATCAE CCTCTAITCT TTGTTCTTAA AITGCCCAGA CTTGTCGTTC AIAACTTCCA CTTACGGCTT CTCAAATCAE CCTCTAITCT ITGTTCTTAA ATTGCCCAGA CTTGTCGTTC AIAACTTCCA

CAAGAAATC TAAAICTAAI AATAAIAAIT ITACGGCTAI TGAACAAATT GAATAITTI CACCCAAACG CAMGAAATC TAAATCTAAI AATAATAAIT ITACGGCTAT TGACAAATT GATTATTT CACCCAAACG

TGTTTTTTI TAAAAAATI ITACACCCCA CGTGGTCATA CAAGTCTGA CGCTTGCAGT CAACAGATTA TGTITITIT IAAAAAAGTI ITACACCCCA CGTGGTCATA CAAGTCTGA CGCTIGCAGI CAACAGATTA

TITGIAIGIT TGIAGGGACA AAAGGITCT GGAGATAAT GGTACGITGI CAAGCAAGT CGCTITCTAG IAIGIATGIT TGIAGGGACA AAGGITTCI GGAGATAAI GGTACGITGI CAAGCAAGTG GGCTITCTAG

TGCACCCATG TAATCGACA GGTGCCTCGG AATCTGTCTA TAAAIACCTG AGAATGGA CTCAACTTTC TGCAECCATE TAATCGACA GGTGCCTCTG AAICTGTCTA TAAAIACCTG AGAAATGGA CTCAGCTTCC

AICGCTCAAA ACACCAAACT TGTTATTACA AGAAITTTT TTGCTTTTTG CACCAAAAGA ACACAAAAAI G AICGCTCAAA ACAACAAACT TCTTACTACA AGAATTTTTI TIGCTTTTTG CACCAAAAGA ACAGAAAAAI G
\end{abstract}

Fig. 2. An alignment of the sequence of $P_{A S S I}$ obtained by a genome walking kit and 5' rapid amplification of cDNA ends PCR of the agarwood sesquiterpene synthase 1 gene. The two fragments can well match, except a 98 bp segment in the $P_{A S S I}$ sequence.

out, and the same result was obtained, which verified the correctness of the cloned sequence (Fig. 1E). To confirm the correctness of the promoter sequence, 5' RACE PCR was performed, and a fragment of $915 \mathrm{bp}$ was amplified. Sequence alignment showed that the two fragments can well match, except for a 98 bp segment missing (Fig. 2). The BLAST in NCBI (https://www.ncbi.nlm.nih.gov/) did not find its homologous sequence, which shows it is an unpublished promoter sequence.

The isolated sequences were searched for the putative cis-acting elements using the Signal Scan program (https:// www.dna.affrc.go.jp/database/). Three putative TATA box sequences were found at $-92 \mathrm{bp}$ (TATAAAT), -776 bp (TATATAT), -923 bp (TATAAAT), respectively, and the putative CAAT box sequences was located at $-609 \mathrm{bp}$ (GCCAAT). Except for these basic functional elements, some cis-elements related to stress-induced regulators in the ASS1 promoter were also found, including five W-boxes (core sequence TGAC or GTCA), six E-boxes (CANNTG), and some MYB, MYC2, DOF, and ABRE recognized elements (Fig. 3). All the regulators are involved in plant defense and stress responses, suggesting the promoter region of $A S S 1$ may respond to a variety of environmental signals. All the cis-elements are summarized in Table 1.

We observed that the ASS1 promoter drove GUS expression ubiquitously in different tissues or organs including the whole seedling, mature leaves, flowers, and stems (Fig. 4), but not in seeds and siliques. 
XU et al.

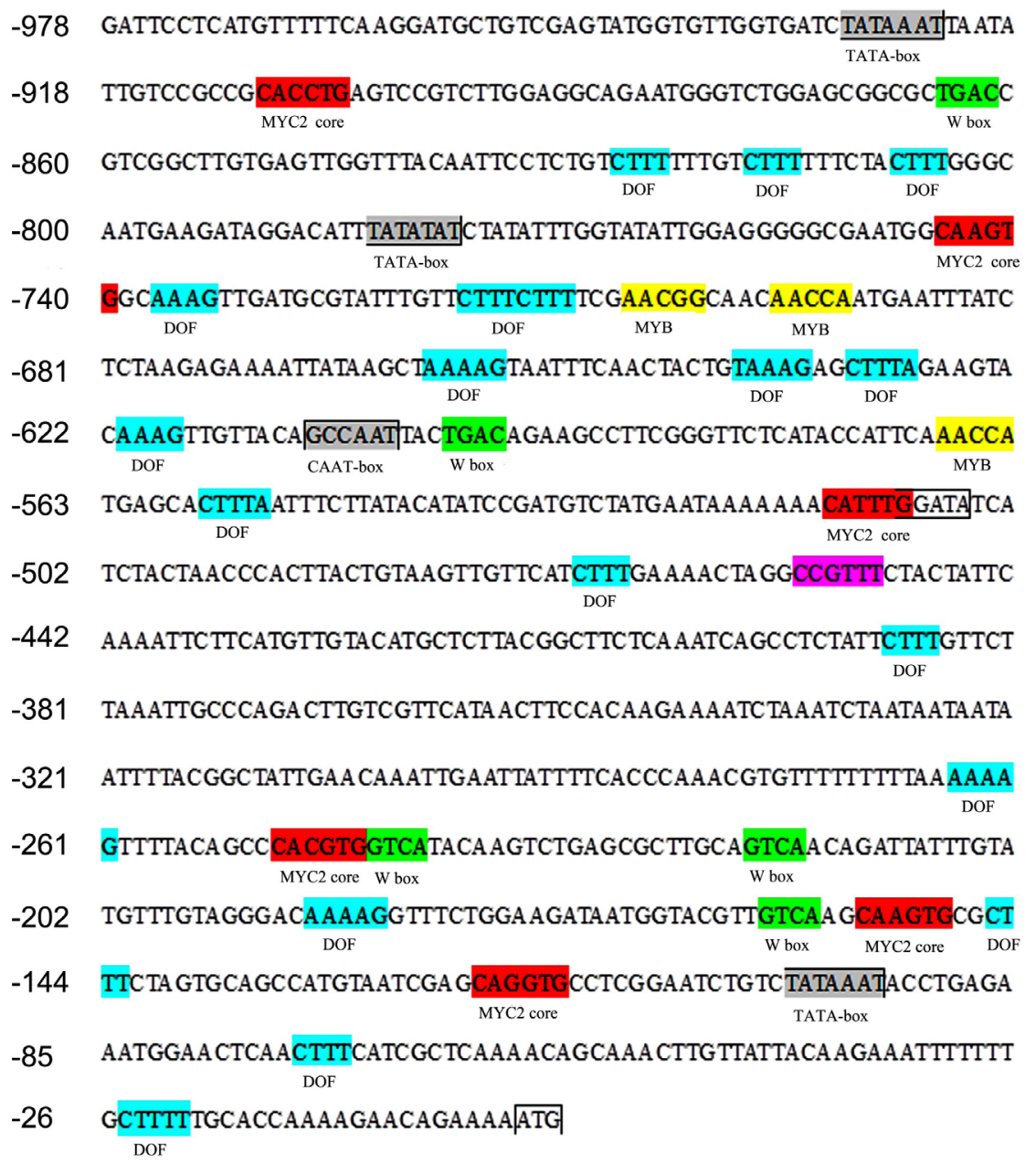

Fig. 3. Characterization and sequence analysis of the agarwood sesquiterpene synthase 1 (ASS1) promoter. A 978 bp ASS1 promoter fragment was cloned and analyzed for its feature motifs by PLACE (https://www.dna.affrc.go.jp/database/). Nucleotide sequences and potential cis-acting elements are marked by different colors: gray - TATA and CAAT boxes; red - cis-acting elements that are recognized by the transcription factor MYC2; green - W-box that is specifically recognized by the transcription factor WRKY; blue - cis-acting elements that are recognized by the transcription factor DOF; yellow - cis-acting elements that are recognized by the transcription factor MYB.

Table 1. Putative cis-acting regulatory elements in the agarwood sesquiterpene synthase 1 promoter: the core sequences and their positions.

\begin{tabular}{lll}
\hline Cis-elements & Sequence & Position \\
\hline ABRE motif & ACGTG & $814,730,698$ \\
DOF motif & AAAG & $242,320,340,358,715,966$ \\
E-box & CANNTG & $71,234,464,729,825,859$ \\
MYB motif & AACCA/AACGG/GGATA & $410,273,469$ \\
MYC motif & CANNTG & $71,234,464,729,825,859$ \\
W-box & TGAC & $114,379,734,758,819$ \\
POLLEN1LELET 52 & AGAAA & $304,633,891,941,973$ \\
\hline
\end{tabular}

From a developmental point of view, cotyledon of the 10 -d-old seedling showed the most intensive staining, while fainter staining was observed in the true leaf. In the flower, sepal showed more intensive GUS staining than petal and anther, and stigma was also stained. It is important that vascular bundle showed the highest steady-state GUS activity in all organs, especially in veins and stem vascular bundle. By controlling the time, it was found the veins were stained first, demonstrating that expression of ASS1 may be vascular bundle specific. Using the transient expression 

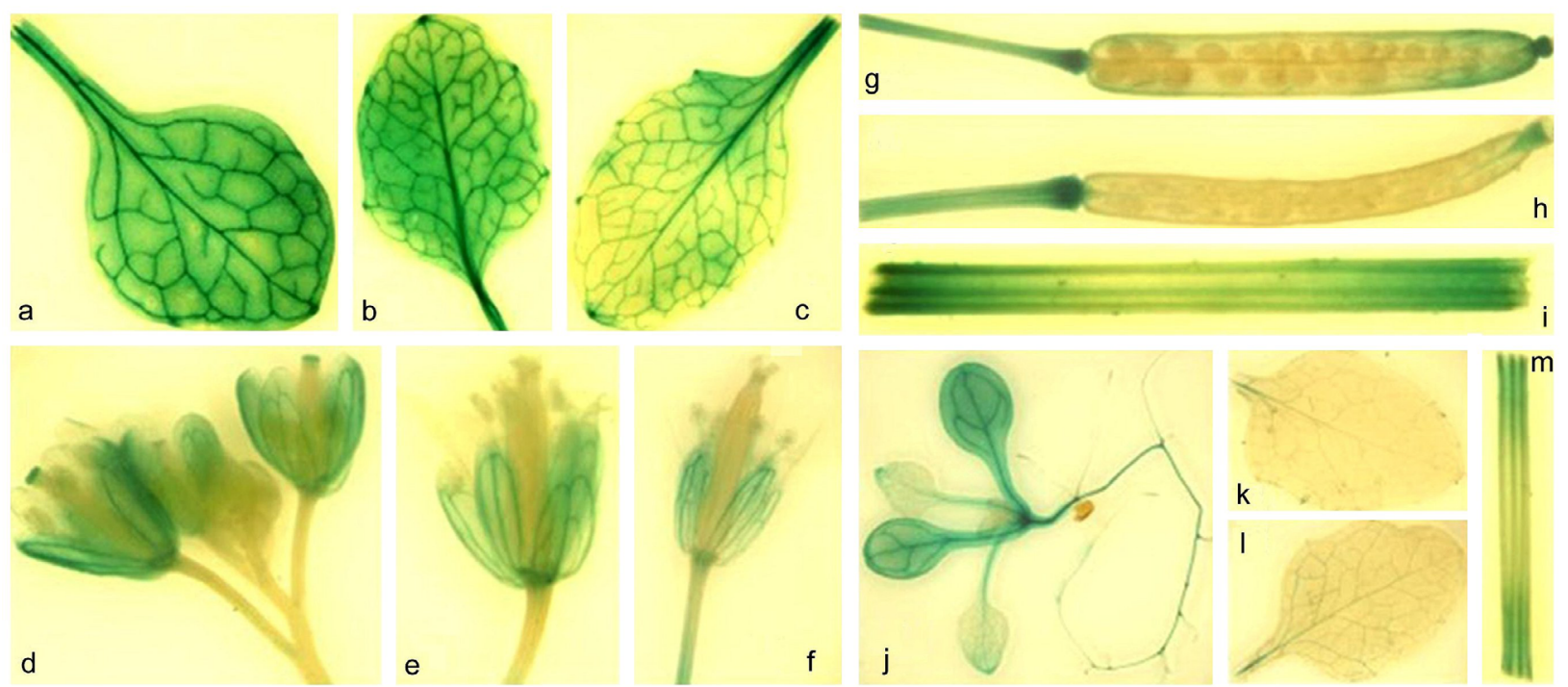

Fig. 4. Tissue specific expression of $\beta$-glucuronidase driven by $P_{A S S I}$ in transgenic Arabidopsis. a-j - stained for $12 \mathrm{~h}$, $\mathrm{k}-\mathrm{m}$ - stained for $8 \mathrm{~h}, \mathrm{a}-\mathrm{c}$ - mature true leaves, $\mathrm{d}-\mathrm{f}$ - flowers, g,h-siliques, i - stem, j - seedling, k, l young leaves, $\mathrm{m}$ - young stem.
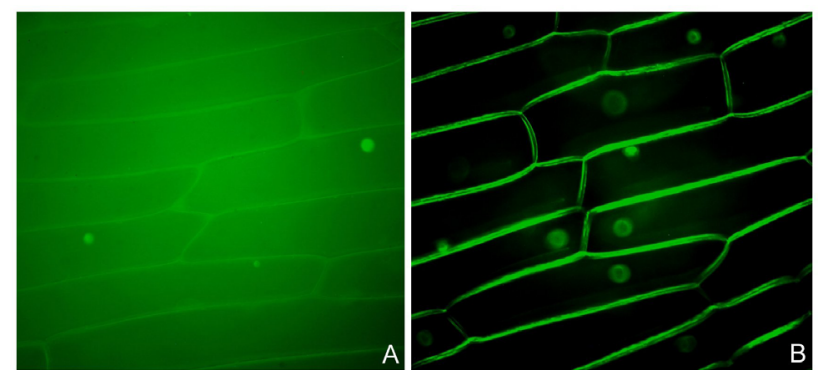

Fig. 5. Localization of green fluorescent protein (GFP) driven by the promoter of agarwood sesquiterpene synthase 1 (ASS1) in cells. Transient expression of the $P_{A S S I}-G F P$ in onion epidermis: $A$ - fluorescence of the empty green fluorescent protein vector, $B$ - fluorescence of the $P_{A S S I}$-GFP.

system in onion epidermis, it was found that ASS1 was localized to nucleus and plasma membranes (Fig. 5). ASS1 mRNA accumulation in suspension-cultured A. sinensis cells is induced by MeJA and a mechanical wound (Xu et al. 2013, 2016). Here, it was also observed that the GUS activity was rapidly stimulated by the mechanical wound, as shown in Fig. $6 \mathrm{~A}$, the GUS staining was deepest around the wound, and become lighter farther from the wound location. Similarly, in three-year-old A. sinensis tree, ASS1 was almost silent in the healthy sample, but it was activated after mechanical cut (Fig. 6C), and its expression sharply rose almost 200 -fold during $1.5 \mathrm{~h}$ (Fig. $6 B$ ).

\section{Discussion}

A. sinensis is the only genuine plant resource for producing agarwood in China and only wounded tree can produce agarwood. The sesquiterpene is one of the most important components of agarwood; its biosynthesis is catalyzed by sesquiterpene synthase. We have previously identified

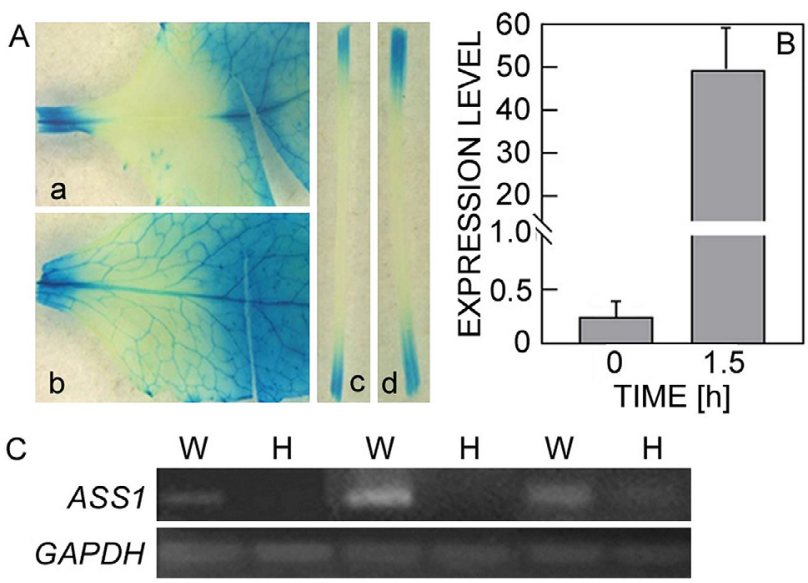

Fig. 6. Mechanical wounding induced expression of $\beta$-glucuronidase driven by $\mathrm{P}_{A S S I}$ in Arabidopsis and expression of agarwood ASS1 in three-year-old Aquilaria sinensis. A - highly expressed GUS around the wound. $B$ - real-time PCR analyzes of ASS1 expression in healthy and wounded three-year-old Aquilaria sinensis trees. Each value is a mean $\pm \mathrm{SE}$ of three independent biological determinations. $C$ - semi-quantitative PCR test of expression of ASS1 in healthy and wounded threeyear-old $A$. sinensis trees; $\mathrm{H}$ - healthy plant, $\mathrm{W}$ - wounded plant.

a sesquiterpene synthase ASS1 from $A$. sinensis, which is responsible for the biosynthesis of sesquiterpenes $\delta$-guaiene, $\alpha$-guaiene, and -humulene (Xu et al. 2013, 2016), and it was a typical inducible gene with expression regulated at the transcriptional level by jasmonic acid and wounding (Chen et al. 2011, Xu et al. 2013). To study the regulation of its expression and the mechanism of woundinduced agarwood sesquiterpenes formation, we cloned and functionally characterized the promoter of $A S S 1$ to understand its expression in different tissues and response to wound treatment.

Sequence analysis showed that the promoter $P_{A S S 1}$, which we cloned, had typical promoter characteristics and 
contained TATA box and CAAT box. In addition, several types of cis-acting elements, including the W-box, DOFbinding motifs, and MYC2-binding motifs, known to be involved in plant defense and stress responses (Pandey and Somssich 2009, Dubos et al. 2010, Kazan and Manners 2013, Noguero et al. 2013), were also found in this promoter region (Fig. 3 and Table 1). It is possible that they may be somehow involved in the regulation of ASS1 expression.

Previous studies have shown that the expression of terpenoid synthase has obvious temporal and spatial specificity (Chen et al. 2003, Yu et al. 2008a,b, Li and Fan 2011). The GUS expression in transgenic Arabidopsis showed that by the promoter of $A S S 1$ driven GUS was distributed in different tissues or organs, including seedling, mature leaf, flower, and stem (Fig. 4). Meanwhile, it is necessary to note that vascular bundles showed the highest steady-state GUS activity no matter what organ they were, demonstrating that expression of ASS1 may be vascular bundle specific. Studying the subcellular localization is a prerequisite for analyzing the correct function of the gene. Further, through the transient expression in onion epidermis, we showed that GFP driven by the promoter of ASS1 was predominantly expressed in nucleus and plasma membranes (Fig. 5). In general, sesquiterpenes are $\mathrm{C} 15$-terpenes predominantly derived from precursors of the cytosolic mevalonate pathway, however, some wild tomato species contain a sesquiterpene pathway that is confined to the chloroplast, and in some cultivated tomato, sesquiterpene synthase is localized in plastids (Sallaud et al. 2009). Shen et al. (2000) identified a sesquiterpene synthase containing $\mathrm{N}$-terminal signal peptide, possibly located in maize chloroplasts, while some sesquiterpene synthase in snapdragon flowers (Nagegowda et al. 2008), strawberry (Aharoni et al. 2004), and Actinidia deliciosa (Nieuwenhuizen et al. 2009) localize in the cytoplasm. These studies indicated that sesquiterpene synthases have different locations due to different functions in plants; their localizations in cells may be affected by the distribution of their substrates (Lange and Ahkami 2013).

As we have shown, $A S S 1$ is a typical inducible gene and agarwood sesquiterpenes can only be formed after injury (Xu et al. 2013, 2016). Here, by GUS staining, we can intuitively see that the expression of $A S S 1$ is induced by mechanical wounding (Fig. 6A). Fluorescence qPCR and semi-qPCR results showed that $A S S 1$ expression was not detected in healthy tissues (Fig. 6C), but increased almost 200 -times after $1.5 \mathrm{~h}$ of mechanical wounding (Fig. $6 B$ ).

In conclusion, we cloned ASS1 promoter and identified its function, localization, and expression. It was predominantly localized in the nucleus and plasma membranes of the cells and also in vascular bundle tissues of different organs. It is an inducible promoter with significant wound-inducible expression profile. These basic characteristics will lay a foundation for further study of its regulatory mechanisms.

\section{References}

Agrawal, A.A.: Induced responses to herbivory and increased plant performance. - Science 279: 1201-1202, 1998.

Aharoni, A., Giri, A.P., Verstappen, F.W.: Gain and loss of fruit flavor compounds produced by wild and cultivated strawberry species. - Plant Cell 16: 3110-3131, 2004.

Arimura, G., Ozawa, R., Shimoda, T., Nishioka, T., Boland, W., Takabayashi, J.: Herbivory-induced volatiles elicit defence genes in lima bean leaves. - Nature 406: 512-515, 2000.

Bang, S.W., Park, S.H., Jeong, J.S., Kim, Y.S., Jung, H., Ha, S.H., Kim, J.K.: Characterization of the stress-inducible OsNCED3 promoter in different transgenic rice organs and over three homozygous generations. - Planta 237: 211-224, 2013.

Chen, F., Tholl, D., D'Auria, J.C., Farooq, A., Pichersky, E., Gershenzon, J.: Biosynthesis and emission of terpenoid volatiles from Arabidopsis flowers. - Plant Cell 15: 481-494, 2003.

Chen, H.Q., Yang, Y., Xue, J., Wei, J.H., Zhang, Z., Chen, H.J.: Comparison of compositions and antimicrobial activities of essential oils from chemically stimulated agarwood, wild agarwood and healthy Aquilaria sinensis (Lour.) Gilg trees. Molecules 16: 4884-4896, 2011.

Chen, H.Q., Wei, J.H., Yang, J.S., Zhang, Z., Yang, Y.: Chemical constituents of agarwood originating from the endemic genus Aquilaria plants. - Chem. Biodiv. 9: 236-250, 2012.

De Moraes, C.M., Lewis, W.J., Pare, P.W., Alborn, H.T., Tumlinson, J.H.: Herbivore-infested plants selectively attract parasitoids. - Nature 393: 570-573, 1998.

Duan, L.X., Chen, T.L., Li, M., Chen, M., Zhou, Y.Q., Cui, G.H., Zhao, A.H., Jia, W., Huang, L.Q., Qi, X.: Use of the metabolomics approach to characterize Chinese medicinal material Huangqi. - Mol. Plants 5: 376-386, 2012.

Dubos, C., Stracke, R., Grotewold, E., Weisshaar, B., Martin, C., Lepiniec, L.: MYB transcription factors in Arabidopsis. Trends Plant Sci. 15: 573-581, 2010.

Farmer, E.E., Ryan, C.A.: Octadecanoid precursors of jasmonic acid activate the synthesis of wound-inducible proteinase inhibitors. - Plant Cell 4: 129-134, 1992

Gomi, K., Yamasaki, Y., Yamamoto, H., Akimitsu, K.: Characterization of a hydroperoxide lyase gene and effect of $\mathrm{C}_{6}$-volatiles on expression of genes of the oxylipin metabolism in Citrus. - J. Plant Physiol. 160: 1219-1231, 2003.

Hashimoto, K., Nakahara, S., Inoue, T., Sumida, Y., Takahashi, M.: A new chromone from agarwood and pyrolysis products of chromone derivatives. - Chem. Pharm. Bull. 33: 50885091,1985

He, J., Giusti, M.M.: Anthocyanins: natural colorants with health-promoting properties. - Annu. Rev. Food Sci. 1: 163$187,2010$.

Hernandez-Garcia, C.M., Finer, J.J.: Identification and validation of promoters and cis-acting regulatory elements. - Plant Sci. 217-218: 109-119, 2014.

Himmelbach, A., Liu, L., Zierold, U., Altschmied, L., Maucher, H., Beier, F., Müller, D., Hensel, G., Heise, A., Schützendübel, A., Kumlehn, J., Schweizer, P.: Promoters of the barley germin-like GER4 gene cluster enable strong transgene expression in response to pathogen attack. - Plant Cell 22: 937-952, 2010.

Hwang, S.H., Lee, I.A., Yie, S.W., Hwang, D.J.: Identification of an OsPR10a promoter region responsive to salicylic acid. Planta 227: 1141-1150, 2008.

Ito, M., Okimoto, K., Yagura, T., Honda, G.: Induction of sesquiterpenoid production by methyl jasmonate in Aquilaria sinensis cell suspension culture. - J. essential Oil Res. 17: 175-180, 2005. 
Itoh, T., Tabata, Y., Widjaja, E., Mulyaningsih T., Parman, H.W.: Structure and artificial induction of aloe wood. - IAWA J. 23: 466-467, 2002.

Kazan, K., Manners, J.M.: MYC2: the master in action. - Mol. Plants 6: 686-703, 2013.

Kessler, A., Baldwin, I.T.: Plant responses to insect herbivory: the emerging molecular analysis. - Annu. Rev. Plant Biol. 53: 299-328, 2002.

Kroymann, J.: Natural diversity and adaptation in plant secondary metabolism. - Curr. Opin. Plant Biol. 1: 246-251, 2011.

Kumeta, Y., Ito, M.: Characterization of $\delta$-guaiene synthases from cultured cells of Aquilaria, responsible for the formation of the sesquiterpenes in agarwood. - Plant Physiol. 154: 19982007, 2010.

Lange, B.M., Ahkami, A.: Metabolic engineering of plant monoterpenes, sesquiterpenes and diterpenes-current status and future opportunities. - Plant Biotechnol. J. 11: 169-196, 2013.

Li, R.H., Fan, Y.P.: Molecular cloning and expression analysis of a terpene synthase gene, HcTPS2, in Hedychium coronarium. - Plant mol. Biol. Rep. 29: 35-42, 2011.

Liu, P.W., Zhang, X.L., Yang, Y., Sui, C., Xu, Y.H., Wei, J.H.: Interxylary phloem and xylem rays are the structural foundation of agarwood resin formation in the stems of Aquilaria sinensis. - Trees 33: 533-542, 2019.

Liu, Y.Y., Chen, H.Q., Yang, Y., Zhang, Z., Wei, J.H., Meng, H.: Whole-tree agarwood-inducing technique: an efficient novel technique for producing high-quality agarwood in cultivated Aquilaria sinensis trees. - Molecules 18: 3086-3106, 2013.

Lou, Y.G., Ma, B., Cheng, J.A.: Attraction of the parasitoid Anagrus nilaparvatae to rice volatiles induced by the rice brown planthopper Nilaparvata lugens. - J. chem. Ecol. 31: 2357-2372, 2007.

Nagegowda, D.A., Gutensohn, M., Wilkerson, C.G., Dudareva, N.: Two nearly identical terpene synthases catalyze the formation of nerolidol and linalool in snapdragon flowers. Plant J. 55: 224-239, 2008.

Nieuwenhuizen, N.J., Wang, M.Y., Matich, A.J., Green, S.A., Chen, X.Y., Yauk, Y.K., Beuning, L.L., Nagegowda, D.A., Dudareva, N., Atkinson, R.G.: Two terpene synthases are responsible for the major sesquiterpenes emitted from the flowers of kiwifruit (Actinidia deliciosa). - J. exp. Bot. 60: 3203-3219, 2009.

Noguero, M., Atif, R.M., Ochatt, S., Thompson, R.D.: The role of the DNA-binding One Zinc Finger (DOF) transcription factor family in plants. - Plant Sci. 209: 32-45, 2013.

Okudera, Y., Ito, M.: Production of agarwood fragrant constituents in Aquilaria calli and cell suspension cultures. Plant Biotechnol. 26: 307-315, 2009.

Pandey, S.P., Somssich, I.E.: The role of WRKY transcription factors in plant immunity. - Plant Physiol. 150: 1648-1655, 2009.

Pellegrineschi, A., Reynolds, M., Pacheco, M., Brito, R.M., Almeraya, R., Yamaguchi- Shinozaki, K., Hoisington, D.: Stress-induced expression in wheat of the Arabidopsis thaliana DREB1A gene delays water stress symptoms under greenhouse conditions. - Genome 47: 493-500, 2004.

Penninckx, I.A., Thomma, B.P., Buchala, A., Métraux, J.P., Broekaert, W.F.: Concomitant activation of jasmonate and ethylene response pathways is required for induction of a plant defensin gene in Arabidopsis. - Plant Cell 10: 21032113, 1998.

Peremarti, A., Twyman, R.M., Gomez-Galera, S., Naqvi, S.,
Farre, G., Sabalza, M., Miralpeix, B., Dashevskaya, S., Yuan, D., Ramessar, K., Christou, P., Zhu, C., Bassie, L., Capell, T. Promoter diversity in multigene transformation. - Plant mol. Biol. 73: 363-378, 2010

Persoon, G.A., van Beek, H.H.: Growing 'the wood of the gods': agarwood production in southeast Asia. Smallholder tree growing for rural development and environmental service. Adv. Agroforest. 5: 245-262, 2008.

Pichersky, E., Gershenzon, J.: The formation and function of plant volatiles: perfumes for pollinator attraction and defense. - Curr. Opin. Plant Biol. 5: 237-243, 2002.

Pojanagaroon, S., Kaewrak, C.: Mechanical methods to stimulate aloes wood formation in Aquilaria crassna Pierre Ex H. LEC. (Kritsana) trees. - ISHS Acta Hort. 676: 161-166, 2005.

Sallaud, C., Rontein, D., Onillon, S., Jabes, F., Duffe, P., Giacalone, C., Thoraval, S., Escoffier, C., Herbette, G., Leonhardt, N., Causse, M., Tissier, A.: A novel pathway for sesquiterpene biosynthesis from Z,Z-farnesyl pyrophosphate in the wild tomato Solanum habrochaltes. - Plant Cell 21: 310-307, 2009.

Shen, B.Z., Zheng, Z.W., Dooner, H.K.: A maize sesquiterpene cyclase gene induced by insect herbivory and volicitin: characterization of wild-type and mutant alleles. - Proc. nat. Acad. Sci. USA 97: 14807-14812, 2000.

Turlings, T.C., Tumlinson, J.H., Lewis, W.J.: Exploitation of herbivore-induced plant odors by host-seeking parasitic wasps. - Science 250: 1251-1253, 1990

Xu, Y.H., Zhang, Z., Wang, M.X., Wei, J.H., Chen, H.J., Gao, Z.H., Sui, C., Luo, H.M., Zhang, X.L., Yang, Y., Meng, H., Li, W.L.: Identification of genes related to agarwood formation: transcriptome analysis of healthy and wounded tissues of Aquilaria sinensis. - BMC Genomics 14: 227, 2013.

Xu, Y.H., Liao, Y.C., Zhang, Z., Liu, J., Sun, P.W., Gao, Z.H., Sui, C., Wei, J.H.: Jasmonic acid is a crucial signal transducer in heat shock induced sesquiterpene formation in Aquilaria sinensis. - Sci. Rep. 6: 21843, 2016.

Yamasaki, Y., Kunoh, H., Yamamoto, H., Akimitsu, K.: Biological roles of monoterpene volatiles derived from rough lemon (Citrus jambhiri Lush) in citrus defense. - J. gen. Plant Pathol. 3: 168-179, 2007.

Yi, N., Oh S.J., Kim, Y.S., Jang, H.J., Park, S.H., Jeong, J.S., Song, S.I., Choi, Y.D., Kim, J.K.: Analysis of the Wsi18, a stress-inducible promoter that is active in the whole grain of transgenic rice. - Transgenic Res. 20: 153-163, 2011.

Yu, F.N., Okamto, S., Nakasone, K., Adachi, K., Matsuda, S., Harada, H., Misawa, N., Utsumi, R.: Molecular cloning and functional characterization of alpha humulene synthase, a possible key enzyme of zerumbone biosynthesis in shampoo ginger (Zingiber zerumbet Smith). - Planta 227: 1291-1299, 2008a.

Yu, F., Harada, H., Yamasaki, K., Okamoto, S., Hirase, S., Tanaka, Y., Misawa, N., Utsumi, R.: Isolation and functional characterization of a beta-eudesmol synthase, a new sesquiterpene synthase from Zingiber zerumbet Smith. FEBS Lett. 582: 565-572, 2008b.

Zhou, M.L., Zhu, X.M., Shao, J.R., Tang, Y.X., Wu, Y.M.: Production and metabolic engineering of bioactive substances in plant hairy root culture. - Appl. Microbiol. Biotechnol. 90: 1229-1239, 2011.

Zou, C., Sun, K., Mackaluso, J.D., Seddon, A.E., Jin, R., Michael, F., Thomashow, M.F., Shiu, S.H.: Cis-regulatory code of stress-responsive transcription in Arabidopsis thaliana. - Proc. nat. Acad. Sci. USA 108: 14992-14997, 2011. 